\title{
Postulaciones políticas en la novela colombiana La casa de las dos palmas, del escritor Manuel Mejía Vallejo
}

Politics applications in the Colombian novel La casa de las dos palmas, by the writer Manuel Mejía Vallejo

\section{Resumen}

Este artículo está basado en la investigación titulada "Postulaciones políticas en la novela colombiana del siglo XX" de la Universidad Cooperativa de Colombia, sede

Bucaramanga, 2009, que consta de tres apartados: la triple mimesis de Ricoeur en Tiempo y narración, análisis del entorno del autor y difusión de su obra en los ambientes escolares.

La novela La casa de las dos palmas de Manuel Mejía hace un recuento de la realidad sociopolítica del momento, el machismo marcado y el sometimiento de la mujer en Colombia.

La novela es trabajada con estudiantes de noveno grado con el fin de motivarlos a la lectura, teniendo en cuenta el tema, objetivos e indicadores de logros, logros y la evaluación. Los estudiantes con las marionetas de material reciclado dramatizan la obra que han leído y analizado en grupos.

Palabras clave Marionetas, mímesis, narración, novela, violencia política.

Abstract

This article is based on the research "Political proposals in the Colombian novel of the 20th century" of Universidad Cooperativa de Colombia, Bucaramanga, 2009, which consists of three parts: the triple mimesis of Ricoeur in time and narrative, the analysis

of author's environment and the dissemination of his work in school environments.

The novel La Casa de las dos palmas written by Manuel Mejia makes reference to the socio-political reality of this time, the marked machismo and the subjugation of women in Colombia.

The novel is worked with ninth grade students in order to motivate them to reading, taking into account theme, objectives, and indicators of achievements, accomplishments and assessment. Students with recycling-material puppets dramatize the work which they have read and analyzed in groups.

Keywords Puppets, mimesis, narrative, novel, political violence.

\section{Liliana-Andrea Sandoval*}

Claudia-Marcela Sierra ${ }^{* *}$

Docente que avala el artículo:

Jairo Castro Neira ${ }^{\text {***\% }}$

Recibido: 4 de marzo del 2011

Aprobado: 18 de abril del 2011
Cómo citar este artículo: Sandoval, LilianaAndrea y Sierra, Claudia-Marcela (2011) "Postulaciones políticas en la novela colombiana La casa de las dos palmas, del escritor Manuel Mejía Vallejo", en Rastros Rostros, vol. 13, núm. 25, pp. 74-79.

* Estudiante de Licenciatura en Educación Básica con énfasis en Humanidades, Lengua castellana e inglés de la Universidad Cooperativa de Colombia, sede Bucaramanga. Correos electrónicos: liliana.sandoval@campusucc.edu.co, liansannu@hotmail.com

** Estudiante de Licenciatura en Educación Básica con énfasis en Humanidades, Lengua castellana e inglés de la Universidad Cooperativa de Colombia, sede Bucaramanga. Correos electrónicos: claudia.sierra@campusucc.edu.co, marcisierra@hotmail.com

*** Licenciado en Idiomas de la Universidad Industrial de Santander. Magíster en Semiótica (Tesista) de la Universidad Industrial de Santander. Docente de la Universidad Cooperativa de Colombia, sede Bucaramanga. Correo electrónico:jairo.castro@campusucc.edu.co 


\section{Introducción}

C omo parte de la formación académica de los nuevos educadores, el programa de Licenciatura en Educación Básica con Énfasis en Humanidades, lengua castellana e inglés de la Universidad Cooperativa de Colombia, desarrolla una investigación titulada "Postulaciones políticas en la novela colombiana del siglo XX", cuya fase final abarcó un corpus de novelas correspondientes a la literatura de la violencia urbana colombiana durante la última mitad del siglo XX.

El presente artículo es fruto del trabajo desarrollado, y está dedicado al análisis de la obra de uno de los más brillantes exponentes de este tipo de literatura, Manuel Mejía, eligiendo para su análisis la novela La casa de las dos palmas.

La extensa obra de Manuel Mejía Vallejo ha contribuido a acrecentar el patrimonio literario, tanto de Antioquia, como de Colombia y de Latinoamérica, con sus valiosos aportes como novelista, cuentista, poeta, crítico, ensayista, prologuista, editor, profesor, periodista, conferencista y promotor de certámenes literarios, y es a partir de dichas postulaciones que resulta significativo adentrarse en el estudio de esta obra en el aula de clase, como herramienta pedagógica en busca del desarrollo crítico de los estudiantes, porque la experiencia ha demostrado que es posible comprometer a profesores y estudiantes en esquemas de formación que exijan más trabajo y reflexión que los esquemas tradicionales (Fecode, 1996, p. 1).

La investigación consta de tres capítulos, el primero de los cuales toma como referente conceptual a Ricoeur (2009) y Cárdenas (2008), notorios en el desarrollo de un discurso literario que establece su dimensión referencial en su significado dinámico y creativo, en la imaginación que introduce como revelación de lo real, de la verdad, de hechos que acontecieron y que marcaron la historia política del país.

El segundo capítulo corresponde al análisis del entorno del autor, hecha a partir de reflexiones y comentarios con tiempo y espacio suficientes, miradas panorámicas que omiten la consideración de las figuras individuales y pueden uniformar el paisaje sin detenerse en sus variedades y que desembocan en un en intrincado contexto, que se hace preciso recoger, analizar e incorporar a nuestra reflexión actual sobre la literatura y, en general, sobre la historia cultural del país.

El tercer apartado está destinado a la difusión de la obra en los ambientes escolares, en la motivación que requieren los estudiantes de noveno grado de la educación básica para apropiarse de esta riqueza cultural, poniendo en escena a través de las marionetas la creación del autor.

\section{Metodología}

De acuerdo con la teoría del relato, se toma como referente la propuesta de la triple mímesis de Paul Ricoeur en Tiempo y narración (Silva, 2005, pp. 167-205), que interrelaciona los momentos de la prefiguración, la configuración y la refiguración del relato.

Este enfoque metodológico se caracteriza por la flexibilidad en cuanto al empleo de técnicas de recolección de datos e información (observación, análisis, entre los más significativos), para hacer uso de técnicas adicionales. En la etapa inicial de la investigación se desarrolla un proceso de observación, para identificar el lenguaje, las formas de vida, el entorno político y la cultura, entre otros y en la segunda etapa se analizan diversos tópicos, tanto de la obra, como del contexto y la realidad nacional.

A lo largo de obra Mejía Vallejo, desde lo prefigurado se presenta la realidad social del momento, el machismo marcado del hombre colombiano y el sometimiento de la mujer (Zoraida Vélez, Evangelina y Lucía Herreros e Isabel), tanto en el orden social, político y religioso, que la lleva a asumir roles superfluos a la sombra del hombre, perdiendo su autoestima al sacrificar su identidad integral, además de ser condenadas al señalamiento y al ostracismo social.

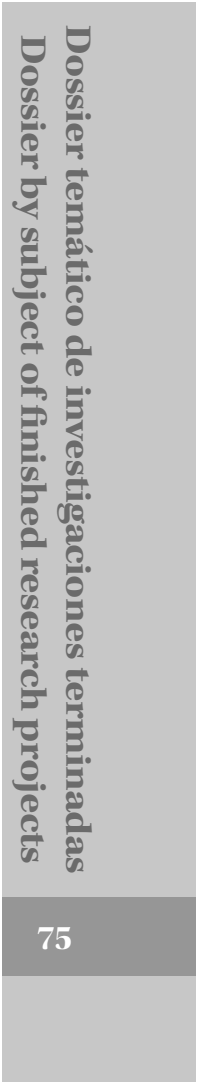

Revista Rastros Rostros - Volumen 13, Número 25 - enero-junio 2011 
El machismo se manifiesta en la actitud de Efraín Herreros, personaje en torno al cual gira la historia de la novela, al arrastrar a su familia, compuesta por esposa, hijos, hermanos y cuñada a la búsqueda de un lugar idóneo en las montañas antioqueñas, de donde es oriundo el escritor, para fundar un pueblo que será sólo de su familia.

Mejía Vallejo nace en el municipio de Jericó (Antioquia) en 1923, territorio que para la época sufría los embates de la violencia política (liberalconservadora) de los años veinte y treinta. Escritor y periodista, egresado de la Universidad Pontificia Bolivariana y del Instituto de Bellas Artes de Medellín, es colaborador del periódico El Sol y creador del grupo "La Tertulia" con Gonzalo Restrepo Jaramillo y Jaime Sanín. Sus convicciones políticas le llevaron a exilarse durante nueve años (1948-1957) en países como Venezuela, Guatemala, Honduras y El Salvador, donde escribe las obras La tierra éramos nosotros (1945) y Al pie de la ciudad (1958). A su regreso al país da rienda suelta a su imaginación y complementa su maravillosa obra literaria con El día señalado (1964, premio Nadal), Aire de tango (1973), Las muertes ajenas (1979, mención especial del Premio Casa de las Américas), Tarde de verano (1981), Y el mundo sigue andando (1984), La sombra de tu paso (1987) y La casa de las dos palmas (1988), por la cual recibe el premio Rómulo Gallegos (Peña, 1997, p. 44).

El autor muestra a través de la novela La casa de las dos palmas la idiosincrasia del colombiano en la primera mitad del siglo XX, resultado de un conflicto político y el abandono al que se ve sometido, razón por la cual vive en divorcio permanente con el Estado, el derecho y la realidad social, que se aprecia en la manera como están reguladas las diferentes instituciones sociales, la familia, las relaciones laborales y la organización de las comunidades.

A través de sus personajes, la familia Herreros, el autor nos lleva por los caminos de la colonización antioqueña. En cada capítulo se reflejan los avatares por los cuales tuvieron que atravesar los llamados colonizadores antioqueños, la peregrinación por tierras inhóspitas en medio de la dura montaña, las inclemencias del clima, las enfermedades y, en fin, todos y cada uno de los obstáculos que el terreno propuso a quienes se atrevieron a abrirse camino por tierras sin dueño, para fundar lo que ahora conocemos como un raza pujante y hondamente comprometida con su propio desarrollo.

Mediante el uso de múltiples figuras literarias, se presenta de una forma abierta, sencilla y entretenida la estructura de las familias antioqueñas, el poder del padre en las decisiones de los miembros de la familia, que hacen emular al "pater familia" de la cultura greco-romana. Asimismo resalta la importancia del papel de la mujer en la formación de los hijos y el mantenimiento del hogar, pues de ella dependía la educación en una edad temprana y la armonía de la familia, por lo general numerosa.

Si bien el libro se centra en la historia de los Herreros, refleja a través de ellos, de sus conflictos internos, las ganas de vivir y de edificarse de toda una generación que, respetando la figura patriarcal, quiere igualmente crear su propio camino, sus propias ideas y de las mujeres que llegan a la vida de los hermanos Herreros, para tratar de sobrellevar aquella maldición, una maldición que no sabremos si es la representación de la vida misma y el querer del hombre por cambiar el entorno en el cual le correspondió vivir y apoderarse cada día de parte de ese mundo que fuera puesto para la conquista del hombre.

El mágico relato permite al lector despertar un interés de principio a fin sobre la vida de los Herreros, sus sueños, frustraciones, deseos, sufrimientos, alegrías y, en fin, es la manifestación misma de cada ser humano puesta en un entorno de paisaje rural en el cual se desarrolla la historia y que en sí refleja el inicio de la vida misma y la tan renombrada "verraquera antioqueña". Sin embargo, debe entenderse que no se trata sólo de mostrar la 
vida en ese lugar de Colombia, sino que en esencia es la fuerza del colombiano puesta al servicio de la supervivencia.

Todo esto sucede en un país que mostraba un total distanciamiento entre los intereses de los "representantes del pueblo" (partidos políticos) y los verdaderos intereses del pueblo (la mayoría, formada por los que viven de su propio trabajo y deben soportar las contracciones de la lucha por el poder), justificada por esa "democracia" que se limita a la realización de elecciones cada periodo de tiempo.

Por ello, uno de los propósitos del análisis "Postulaciones políticas en la novela colombiana La casa de las dos palmas del escritor Manuel Mejía Vallejo” es mostrar la emancipación de la literatura, como una toma de posición valorativa, orientadora y abierta a las transformaciones de la sociedad, el horizonte común de la realidad vivida, de las experiencias y necesidades sociales, sin excluir ninguna de las partes interrelacionadas que la generan, estructurada en torno a la socio crítica (Manuel Mejía Vallejo y la sociedad de su tiempo: la occidentalización de la sociedad colombiana del siglo XX), tiempo y narración (Mejía Vallejo y lo absurdo del destino humano), el siglo XX de la historia de Colombia (el pueblo que no conoce su historia está condenado a repetirla), la novela colombiana del siglo XX, espacios de experiencia (Manuel Mejía Vallejo: escritor), espacios de experiencia de la obra (La casa de las dos palmas: "la torpeza de condenar, de no perdonar"), y el cronotopo de la violencia, viendo cómo el escritor innova y crea, por cuanto modifica los cánones anteriores de la literatura, y al hacerlo padece la influencia de ella y de su época social, a la vez que impone su personalidad.

Tan es así que es el resultado de la incorporación del modelo cultural, hecho síntesis en la sociedad que buscaba la reivindicación de sus derechos, y que el escritor describe con propiedad, las ideas políticas, las luchas sociales, el cuerpo institucional del Estado, así como las diferentes manifestaciones de la sociedad colombiana, impuestas durante la conquista y colonización, así como de los subsiguientes procesos independentistas que culminaron con el establecimiento de los regímenes democráticos que vivimos actualmente, pero que se vieron seriamente amenazados sobre todo en la primera mitad del siglo XX.

Existen también sucesos o cadenas de sucesos que por su extensión y profundidad marcan a generaciones enteras y dejan huellas imborrables que, se quiera o no, entran a formar parte de la identidad social de la época, que se manifiesta en las obras de Mejía Vallejo, a través de La casa de las dos palmas. Seguramente entre los más notables está el fenómeno de la violencia, y dentro de esta línea de análisis las raíces prehispánicas de algunos elementos estructurales del conflicto.

\section{Resultados}

El deseo constante que ha sentido el hombre por conocer el mundo que le rodea, de explicarlo y deducir de él sus principios, sus relaciones, su cultura, su devenir, motiva a los docentes en una época de globalización a fomentar la calidad de la educación desde la lectura. La lectura incrementa la capacidad de comprender su cultura, ayuda en el desenvolvimiento del hombre en todos sus campos, amplía la visión del mundo para interpretar la vida, para relacionarse con su entorno, y es vital para el desarrollo de las personas, la ciencia y la sociedad. El desenvolvimiento personal, profesional y social de un individuo depende de la capacidad de comprensión, interpretación, expresión y recreación de la realidad humana.

Aunado a esa motivación, en el proceso de formación docente que se imparte desde la Universidad Cooperativa de Colombia y que lleva sus propuestas a las aulas en establecimientos oficiales de la básica secundaria en Bucaramanga, se pretende implementar nuevas estrategias de motivación hacia la lectura, dando a su vez a conocer la vida y obra de

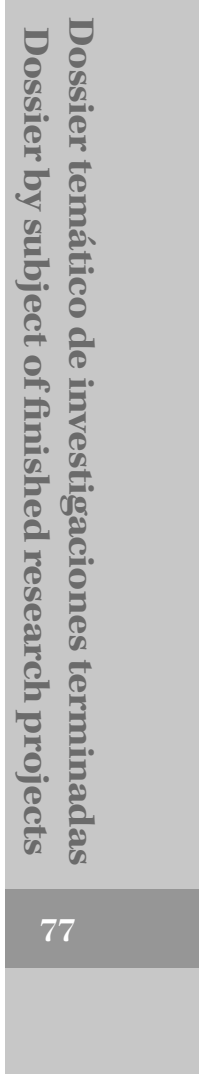

Revista Rastros Rostros - Volumen 13, Número 25 - enero-junio 2011 
importantes escritores colombianos como Manuel Mejía Vallejo y su obra cumbre La casa de las dos palmas, que refleja la historia política de Colombia en la celebración del bicentenario de independencia.

Esa misma motivación que se alimenta desde la universidad es la que como docentes en formación se transmite a los niños, haciendo énfasis en que, cuando la lectura es activa, se comprende y se aprende, y esto implica hacer un procesamiento de la información y emplear una serie de conocimientos, hipótesis y experiencias previas. Asociado a ello está el proceso interno de lectura, puesto que al leer cada uno le está dando al texto un significado propio.

Ante esta realidad, y teniendo en cuenta que se hace necesario motivar a los estudiantes de noveno grado de la básica secundaria de educación pública a adquirir hábitos de lectura, se plantea como metodología de trabajo la representación o dramatización de la obra a través de marionetas, las cuales son elaboradas con material de reciclaje, dado que desde la Universidad se hace énfasis en la protección del medio ambiente y el desarrollo sostenible (Universidad Cooperativa de Colombia, 2011, Visión).

Para tal fin los alumnos se organizan en cinco grupos de trabajo, cada uno de los cuales se encarga del análisis y dramatización de cinco capítulos. Inicialmente se hace un recuento general de la obra para que los estudiantes tengan una idea de cómo se realiza la trama, y a partir de ella se generan una serie de talleres sobre técnicas de lectura, comprensión de lectura, elaboración de argumentos y fabricación de las marionetas.

Esto incluye una parcelación, en la cual se tiene en cuenta: tema, objetivos, indicadores de logros, logros y evaluación, vista esta última como pedagogía de acompañamiento y proceso investigativo, de manera que una profunda reflexión frente a los elementos que intervienen en todo acto educativo compromete a desarrollar propuestas de trabajo desde los propios intereses, recursos y motivaciones educativas.
Teniendo en cuenta que una condición básica del ser humanos es su capacidad para cambiar y aprender, como un sistema abierto, flexible, autoplástico, impredecible y susceptible de experimentar cambios estructurales significativos, la motivación a la lectura está encaminada a la reflexión acerca de los procesos cognitivos, la reflexión a partir de la acción, la aplicación de los aprendizajes, la competencia para resolver problemas y para tomar decisiones.

Esta aplicación de aprendizajes, de competencias para resolver problemas y toma decisiones, es aprovechada para generar conciencia ambiental (la misma que Mejía Vallejo relaciona al destacar la imponencia del paisaje, las corrientes de agua, el verde de la montaña, seriamente afectado por el mal llamado desarrollo y ahora globalización) en la representación de personajes y en la puesta en escena de locaciones.

A través de la puesta en escena de la obra, los niños pueden llegar a asimilar las realidades intelectuales, que sin ellos seguirían siendo exteriores y extrañas para su formación académica. En los momentos pedagógicos actuales se acude a las marionetas como una herramienta de gran interés en procesos de aprendizaje; la implementación de metodologías lúdicas que permiten una participación activa y un disfrute del proceso de aprendizaje refuerzan cualquier actividad de formación curricular.

Se potencia la creatividad, como identidad del ser humano, aprovechada, tanto por los participantes para que interpreten, comprendan y actúen racionalmente en la dramatización de la obra, como también por el orientador o facilitador para lograr motivar y despertar inquietudes en los estudiantes.

Para el éxito de la estrategia pedagógica, es importante resaltar la estrecha relación que debe existir entre el profesor, los participantes, los estudiantes y el ambiente; el profesor no debe limitarse sólo a transmitir u orientar un conocimiento determinado, es necesario que se relacione con sus alumnos para 
conocer sus expectativas, formas de pensar, actuar y para que de esta forma se puedan mejorar los canales de comunicación y los procesos de aprendizaje.

Es necesario motivar al estudiante para que proponga alternativas, exprese sus inquietudes, construya el conocimiento, para que se sienta parte del proceso de aprendizaje. Se debe tener presente que los seres humanos podemos aprender de muchas formas, la utilización de todos los sentidos permite ampliar las alternativas de exploración, por ello es importante generar espacios y momentos que le permitan a los estudiantes expresar sus sentimientos y expectativas, y nada mejor que a través de la dramatización de la novela La casa de las dos palmas.

\section{Conclusiones}

Cualquiera que sea la actitud que tome el niño (estudiante de básica secundaria), ésta proporciona una forma diferente de conocimiento, capaz de dar respuestas a interrogantes, porque el universo en general es la fuente inagotable de conocimientos.

Si partimos del adagio popular que reza "un pueblo que no conoce su historia está condenado a repetirla”, el análisis de la obra de Manuel Mejía Vallejo nos permite hacer un recorrido por la historia de nuestro país, no sólo como un encuentro con el pasado, sino con la posibilidad de entender el presente, conjugando sus múltiples y diversos procesos.
$\mathrm{Su}$ importancia radica en que la obra es una indagación sobre el significado de la vida individual y colectiva de los seres humanos en el transcurso del tiempo, dotado de un pasado común, fundado en ese origen remoto y en la identidad colectiva, porque el acontecimiento aislado es poco significativo y lo que cuenta es el mecanismo que articula un conjunto de acontecimientos, que surgen de las raíces profundas que sostienen las sociedades, las naciones y las culturas y, asimismo, es la disciplina que esclarece el pasado de los individuos: es el saber que devela las raíces sociales del ser humano.

\section{Referencias}

Fecode (1996), “Educación y cultura: la enseñanza de las ciencias sociales”, núm. 40, Bogotá.

Mejía Vallejo, M. (2003), La casa de las dos palmas, Nomos, Bogotá.

Peña Gutiérrez, I. (1997), Manual de la Literatura Latinoamericana, Educar, Bogotá.

Silva Arévalo, E. (2005), "Paul Ricoeur y los desplazamientos de la hermenéutica”, en Revista Teología y vida, vol. XLVI, pp. 167-205, Pontificia Universidad Católica de Chile.

Universidad Cooperativa de Colombia. (2011), "Visión”, en Prospectos Licenciatura en Educación Básica con Énfasis, en Humanidades, lengua castellana e inglés, Bucaramanga.

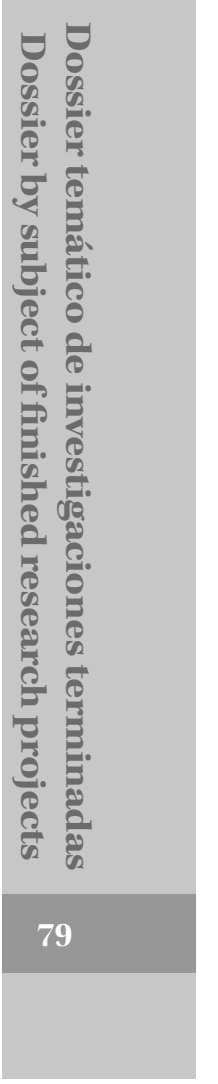

Revista Rastros Rostros - Volumen 13, Número 25 - enero-junio 2011 